\title{
The Size of a Share Must Be Large
}

\author{
László Csirmaz* \\ Mathematical Institute of the Hungarian Academy of Sciences \\ Reáltanoda u. 13-15, Budapest, Hungary, H-1053
}

\begin{abstract}
A secret sharing scheme permits a secret to be shared among participants of an $n$-element group in such a way that only qualified subsets of participants can recover the secret. If any non-qualified subset has absolutely no information on the secret, then the scheme is called perfect. The share in a scheme is the information what a participant must remember. We prove that for each $n$ there exists an access structure on $n$ participants so that any perfect sharing scheme must give some participant a share which is at least about $n / \log n$ times the secret size ${ }^{2}$. We also show that the best possible result achievable by the information theoretic method used here is $\boldsymbol{n}$ times the secret size.
\end{abstract}

Key words. Secret sharing, ideal secret sharing schemes, polymatroid structures, perfect security.

\section{Introduction}

An important issue in secret sharing schemes is the size of the shares distributed to the participants since the security of a system degrades if the amount of the information that must be kept secret increases. The problem of giving bounds on the size of the secret some participant must have has received considerable attention in the last few years, see e.g. [12], [4], [5].

R. M. Capocelli et al. [4] showed that in a certain access structure with four participant the number of the bits some participant must remember is at least 1.5 times the number of bits in the secret. They generalized the construction to any number of participants with the same bound. In [2] and [3] the bound $2-\varepsilon$ was proved. M. van Dijk in [8] proved $\log n$ for a certain access structure on $n$ participants. The method was information-theoretic, namely the results followed by a close examination of the entropy of the information a group of the participants have. The connection between the entropy and matroid-theory was observed by Fujishige [9], and in the context of secret sharing scheme by K. Kurosawa et al. [11]. Here we expand these ideas to our main result:

Theorem 1. For each $n$ there exists an access structure $\mathcal{A}$ on $n$ participants so that any perfect secret sharing scheme assigns a share of length about $n / \log n$ times the length of the secret to some participant.

* This research was supported by OTKA grant no. 1911

${ }^{2}$ All logarithms in this paper are of base 2 . 
We give a construction which shows that apart from the $\log n$ factor, our result is the best possible. That is, the information theoretic method cannot yield a lower bound for the size of the share of the participants better than $n$ times the size of the secret.

Let us call a participant $x$ unimportant if no unqualified group becomes qualified by adapting $x$. Obviously, in any secret sharing scheme the share of an unimportant participant can safely be disregarded, thus $x$ 's share can be considered zero. The following theorem is implicit in [4]:

Theorem 2. In any perfect secret sharing scheme, all important participant must have a share at least as large as the secret itself.

This bound is the best possible, as R. M. Capocelli et al. [4] observed that in any access structure fixing any participant $x$, it is possible to distribute the shares so that $x$ 's share will be of the same length as the secret.

\section{Preliminaries}

In this section we review the technical concepts as well as some earlier results. For a complete treatment of information theory the reader is referred to [6]; its application to secret sharing is explained in details in [4]. For the sake of completeness we repeat here some definitions and lemmas.

\subsection{Information Theoretic Notions}

Given a probability distribution $\{p(x)\}_{x \in X}$ on a finite set $X$, define the entropy of $X, H(X)$ as

$$
H(X)=-\sum_{x \in X} p(x) \log p(x) .
$$

The entropy $H(X)$ is a measure of the average information content of the elements in $X$. It is well known that $H(X)$ is a good approximation to the average number of bits needed to represent the elements of $X$ faithfully. By definition, the entropy is always non-negative.

Given two sets $X$ and $Y$ and a joint probability distribution $\{p(x, y)\}_{x \in X, y \in Y}$ on the Cartesian product of $X$ and $Y$, the conditional entropy $H(X \mid Y)$ of $X$ assuming $Y$ is defined as

$$
H(X \mid Y)=\sum_{y \in Y} p(y) H(X \mid Y=y),
$$

where " $X \mid Y=y$ " is the probability distribution got from $p$ by fixing the value $y \in Y$. The conditional entropy can also given in the form

$$
H(X \mid Y)=H(X Y)-H(Y)
$$

where $Y$ is the marginal distribution. From definition (1) it is easy to see that the $H(X \mid Y) \geq 0$. 
The mutual information between $X$ and $Y$ is defined by

$$
\begin{aligned}
I(X ; Y)= & H(X)-H(X \mid Y)=H(Y)-H(Y \mid X) \\
& =H(X)+H(Y)-H(X Y)
\end{aligned}
$$

and is always non-negative: $I(X ; Y) \geq 0$. This inequality expresses the intuitive fact that the knowledge of $Y$, on average, can only decrease the uncertainty one has on $X$.

Similarly to the conditional entropy, the conditional mutual information between $X$ and $Y$ given $Z$ is defined as

$$
\begin{gathered}
I(X ; Y \mid Z)=H(X \mid Z)-H(X \mid Y Z) \\
=H(X Z)+H(Y Z)-H(X Y Z)-H(Z),
\end{gathered}
$$

and is also non-negative: $I(X ; Y \mid Z) \geq 0$.

\subsection{Secret Sharing Schemes}

In the following individuals will be denoted by small letters: $a, b, x, y$, etc., sets (groups) of individuals by capital letters $A, B, X, Y$, etc., finally collections of groups by script letters $\mathcal{A}, \mathcal{B}$. We use $P$ to denote the set of participants who will share the secret.

An access structure on an $n$-element set $P$ of participants is a collection $\mathcal{A}$ of subsets of $P$ : exactly the qualified groups are collected into $\mathcal{A}$. We shall denote a group simply by listing its members, so $x$ denotes both a member of $P$ and the group which consists solely of $x$. From the context it will always be clear which meaning we are using.

A secret sharing scheme permits a secret to be shared among $n$ participants in such a way that only qualified subsets of them can recover the secret. Secret sharing schemes satisfying the additional property that unqualified subsets can gain absolutely no information about the secret is called perfect as opposed to schemes where unqualified groups may get some information on the secret (e.g. the ramp schemes on [1].

A natural property of the access structures is its monotonicity, i.e. $A \in \mathcal{A}$ and $A \subseteq B \subseteq P$ implies $B \in \mathcal{A}$. This property expresses the fact that if any subset of $B$ can recover the secret then the participants in $B$ can also recover the secret. Also, a natural requirement is that the empty set should not be in $\mathcal{A}$, i.e. there must be some secret at all. Access systems of this type are called Sperner systems, named after E. Sperner who was the first to determine the maximal number of subsets in such a system [13].

Let $P$ be the set of participants, $\mathcal{A}$ be a Sperner system on $P$, and let $S$ be the set of secrets. A secret sharing scheme, given a secret $s$, assigns to each member $x \in P$ a random share from some domain. The shares are thus random variables with some joint distribution determined by the value of the secret $s \in S$. Thus a scheme can be regarded as a collection of random variables, one for the secret, and one for each $x \in P$. The scheme determines the joint distribution of these 
$n+1$ random variables. For $x \in P$ the $x$ 's share, which is (the value of) a random variable, will also be denoted by $x$. For a subset $A$ of participants, $A$ also denotes the joint (marginal) distribution of the shares assigned to the participants in $A$.

Following [4] we call the scheme perfect if the following hold:

1. Any qualified subset can reconstruct the secret, that is, the shares got by the participants in $A$ determine uniquely the secret. This means $H(s \mid A)=0$ for all $A \in \mathcal{A}$.

2. Any non qualified subset has absolutely no information on the secret, i.e. $s$ and the shares got by members of $A$ are statistically independent: knowing the shares in $A$, the conditional distribution of $s$ is exactly the same as its a priori distribution. Translated to information theoretic notions this gives $H(s \mid A)=H(s)$ for all $A \notin \mathcal{A}$.

By the above discussion the entropy of the secret, $H(s)$, can be considered as the length of the secret. Any lower bound on the entropy of $x \in P$ gives immediately a lower bound on the size of $x$ 's share: if $H(x) \geq \lambda H(s)$ then $x$ 's share is at least $\lambda$ times the size of the secret.

\subsection{Polymatroid structure}

Let $Q$ be any finite set, and $B=2^{Q}$ be the collection of the subsets of $Q$. Let $f: \mathcal{B} \rightarrow \mathbf{R}$ be a function assigning real numbers to subsets of $Q$ and suppose $f$ satisfies the following conditions:

(i) $f(A) \geq 0$ for all $A \subseteq Q, f(\emptyset)=0$,

(ii) $f$ is monotone, i.e. if $A \subseteq B \subseteq Q$ then $f(A) \leq f(B)$,

(iii) $f$ is submodular, i.e. if $A$ and $B$ are different subsets of $Q$ then $f(A)+f(B) \geq$ $f(A \cap B)+f(A \cup B)$.

The system $(Q, f)$ is called polymatroid. If, in addition, $f$ takes only integer values and $f(x) \leq 1$ for one-element subsets, then the system is a matroid.

S. Fujishige in [9] observed that having a finite collection of random variables, we will get a polymatroid by assigning the entropy to each subset. The following proposition can also be found in [11].

Proposition 3 By defining $f(A)=H(A) / H(s)$ for each $A \subseteq P \cup\{s\}$ we get a polymatroid.

Proof. We check (i)-(iii) of the definition of the polymatroid. (i) is immediate since the entropy is always non-negative. (ii) follows from (2) by letting $X=B$, $Y=A$. Then $X Y=X \cup Y=X$, i.e.

$$
f(B)-f(A)=H(X Y)-H(Y)=H(X \mid Y) \geq 0 .
$$

Similarly, (iii) follows easily from (3) and from the fact that the conditional mutual information $I(X ; Y \mid Z) \geq 0$. 
Unfortunately, it is not known whether the converse of this proposition holds, i.e. all polymatroids over a finite set can be got as the entropy of appropriately chosen random variables [7]. We shall elaborate on this later.

In our case the random variable $s$, the "secret" plays a special role. By our extra assumption on the conditional entropies containing $s$, we can calculate the value of $f(A s)$ from $f(A)$ for any $A \subseteq P$, see $[4,11]$.

Proposition 4 If the secret sharing scheme is perfect, then for any $A \subseteq P$ we have

if $A \in \mathcal{A}$ then $f(A s)=f(A)$;

if $A \notin \mathcal{A}$ then $f(A s)=f(A)+1$.

Proof. If $A \in \mathcal{A}$ then $A$ is a qualified subset, an thus $H(s \mid A)=0$. By definition, $H(s \mid A)=H(s A)-H(A)$, and the first claim follows.

If $A \notin \mathcal{A}$ then $A$ is an unqualified subset, and then $H(s \mid A)=H(s)$, which yields the second claim.

Now let us consider the function $f$ defined in Proposition 3 restricted to the subsets of $P$. From this restriction we can calculate easily the whole function; and since the extension is also a polymatroid, the restriction will satisfy some additional inequalities.

Proposition 5 The function $f$ defined in Proposition 3 satisfies the following additional inequalities:

(i) if $A \subseteq B, A \notin \mathcal{A}$ and $B \in \mathcal{A}$ then $f(B) \geq f(A)+1$;

(ii) if $A \in \mathcal{A}, B \in \mathcal{A}$ but $A \cap B \notin \mathcal{A}$ then $f(A)+f(B) \geq f(A \cap B)+f(A \cup B)+1$.

Proof. If $A \subseteq B$ then $A s \subseteq B s$, therefore by the monotonicity of $f$ we have

$$
f(A)+1=f(A s) \leq f(B s)=f(B)
$$

which gives (i). Similarly, using the submodularity for the sets $A s, B s$ we get (ii).

The claim of this proposition can be reversed: given any polymatroid $f$ on the subsets of $P$ satisfying (i) and (ii) above and extending $f$ to the subsets of $P \cup\{s\}$ as defined in Proposition 4, we get a polymatroid.

\section{Results}

We start by proving

Theorem6. In any perfect secret sharing scheme, all important participant must have a share at least as large as the secret itself. 
Proof. Suppose an access structure $\mathcal{A}$ is given on the set $P$ of participants, $x \in P$ is an important person shown by $C \subseteq P$, i.e. $C \notin \mathcal{A}$ but $C x \in \mathcal{A}$. Also let us given any perfect secret sharing scheme, and consider the function $f$ defined in Proposition 3. Since $f(x)=H(x) / H(s), f(x) \geq 1$ implies $H(x) \geq H(s)$, i.e. that the (average) size of $x$ 's share must be at least as large as the (average) size of the secret. Thus we have to show only that $f(x) \geq 1$.

Since $C \notin \mathcal{A}$ and $C x \in \mathcal{A}$, by Proposition 5 (i) we have $f(C x) \geq f(C)+1$. $f$ is submodular on the subsets of $P$, so we also have

$$
f(C)+f(x) \geq f(C x)+f(C \cap\{x\})=f(C x)+f(\emptyset)=f(C x)
$$

since $x \notin C$. Combining this with $f(C x) \geq f(C)+1$ we get the desired result.

Theorem 7. For each $n$ there exists an access structure $\mathcal{A}$ on $n$ participants so that any perfect secret sharing scheme assigns a share of length about $n / \log n$ times the length of the secret to some participant.

Proof. Suppose an access structure $\mathcal{A}$, to be defined later, is given on the $n$ element set $P$ of participants. Let $k$ be the largest integer with $2^{k}+k-2 \leq n$. Suppose also that a perfect secret sharing scheme is given, and consider again the function $f$ defined in Proposition 3. We have to find a participant $x \in P$ such that $f(x)$ at least $\left(2^{k}-1\right) / k$ which is approximately equal to $n / \log n$ (for example, it is always between $n / 2 \log n$ and $n / \log n$ ).

We illustrate the construction by an example for $k=2$. Let $a, b, c, d$ be different members of $P$. (Since $2^{k}+k-2=4 \leq n$ there are at least four members in $P$.) Let the sets $a b, c a$, and $c d b$ be minimal sets in the Sperner system $\mathcal{A}$, i.e. none of their proper subsets are in $\mathcal{A}$ (see Figure 1, elements of $\mathcal{A}$ are denoted by full dots).

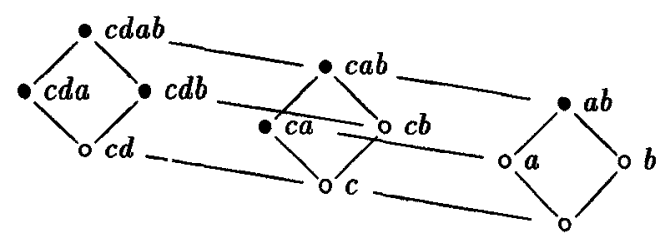

Fig. 1. The case $k=2$

Now consider the following differences:

(i) $f(c d a b)-f(c d)$;

(ii) $f(c a b)-f(c)$;

(iii) $f(a b)-f(\emptyset)$.

Since $c d a b \in \mathcal{A}$ and $c d \notin \mathcal{A}$, by Proposition 5 (i) we have (i) $\geq 1$. We claim that each difference is at least 1 larger than the previous one. To show this, we use 
Proposition 5 (ii) and the submodularity of $f$ as follows. Since $c d b$, and $c a b$ are both in $\mathcal{A}$, but their intersection, $c b \notin \mathcal{A}$, we have

$$
f(c d b)+f(c a b) \geq f(c d a b)+f(c b)+1 .
$$

Applying the submodularity to $c d$ and $c b$ we have

$$
f(c d)+f(c b) \geq f(c d b)+f(c) .
$$

Adding up and rearranging the terms we get

$$
f(c a b)-f(c) \geq f(c d a b)-f(c d)+1
$$

which shows that (ii) $\geq$ (i) +1 .

Similarly, applying Proposition 5 (ii) to $c a$ and $a b$ and the submodularity to $c$ and $a$ we get (iii) $\geq$ (ii) $+1 \geq$ (i) $+2 \geq 3$. Now since $f(a)+f(b) \geq f(a b)$ (by submodularity again) which is $\geq 3$, either $f(a)$ or $f(b)$ must be at least 1.5 , i.e. either $a$ or $b$ must have a share with size $50 \%$ bigger than the size of the secret. This was the main result in [4] using a slightly different access structure.

Now let us turn to the general construction. Let $A$ be a $k$-element set of individuals, and $A=A_{0}, A_{1}, \ldots, A_{2^{k}-1}=\emptyset$ be a decreasing enumeration of all of its subsets so that if $i<j$ then $A_{i} \nsubseteq A_{j}$. Let $B=\left\{b_{1}, b_{2}, \ldots, b_{2^{k}-2}\right\}$ be disjoint from $A$, our set of individuals will be $A \cup B$. Since $k+2^{k}-2 \leq n$ we can pick $A$ and $B$ from $P$. Let $B_{0}=\emptyset$, and in general $B_{i}=\left\{b_{1}, b_{2}, \ldots, b_{i}\right\}$. The minimal elements of the access structure $\mathcal{A}$ will be $U_{i}=A_{i} \cup B_{i}$ for $i=0$, $1, \ldots, 2^{k}-2$. They are pairwise incomparable, i.e. none of them is a subset of the other; this means that they indeed can form the minimal elements in an access structure. To check it, let $i<j$, then $b_{j} \in U_{j}-U_{i}$ (i.e. $U_{j} \nsubseteq U_{i}$ ), and $\emptyset \neq A_{i}-A_{j} \subseteq U_{i}-U_{j}$ (i.e. $U_{i} \nsubseteq U_{j}$ ).

Lemma 8. Under these assumptions, for each $0 \leq i<2^{k}-2$

$$
\left[f\left(B_{i} \cup A\right)-f\left(B_{i}\right)\right]-\left[f\left(B_{i+1} \cup A\right)-f\left(B_{i+1}\right)\right] \geq 1 .
$$

Proof. Just mimic the proof for the case $k=2$. Choosing $X=B_{i} \cup A, Y=$ $B_{i+1} \cup A_{i+1}$, both of them are in $\mathcal{A}$ since $X \supseteq U_{i}$, and $Y=U_{i+1}$, while $X \cap Y=$ $B_{i} \cup A_{i+1} \notin \mathcal{A}$. To see this it is enough to check that for all $j, U_{j}=A_{j} \cup B_{j} \nsubseteq$ $B_{i} \cup A_{i+1}$. Indeed, if $j \leq i$ then $A_{j} \nsubseteq A_{i+1}$; if $j>i$ then $B_{j} \nsubseteq B_{i}$. Therefore by Proposition 5 (ii) we have

$$
f(X)+f(Y) \geq f(X \cup Y)+f(X \cap Y)+1,
$$

or, by rearranging,

$$
\left[f\left(B_{i} \cup A\right)-f\left(B_{i} \cup A_{i+1}\right)\right]-\left[f\left(B_{i+1} \cup A\right)-f\left(B_{i+1} \cup A_{i+1}\right)\right] \geq 1 .
$$

The submodularity of $f$ applied to $X=B_{i} \cup A_{i+1}$ and $Y=B_{i+1}$ gives

$$
f(X)+f(Y) \geq f(X \cup Y)+f(X \cap Y),
$$

i.e. also by rearranging the terms

$$
\left[f\left(B_{i} \cup A_{i+1}\right)-f\left(B_{i}\right)\right]-\left[f\left(B_{i+1} \cup A_{i+1}\right)-f\left(B_{i+1}\right)\right] \geq 0 .
$$

By adding up inequalities (4) and (5) we get the claim of the lemma. 
Lemma 9. $f(A) \geq 2^{k},-1$.

Proof. Note that $f\left(A \cup B_{2^{k}-2}\right)-f\left(B_{2^{k}-2}\right) \geq 1$ by Proposition 5 (i) since $A \in \mathcal{A}$ but $B_{2^{k}-2} \notin \mathcal{A}$. Now adding this to the inequality in Lemma 8 for all $0 \leq i<$ $2^{k}-2$ we get

$$
f\left(B_{0} \cup A\right)-f\left(B_{0}\right) \geq 2^{k}-1,
$$

which, by $B_{0}=\emptyset$, gives the result.

Finally, by iterated application of the submodularity inequality,

$$
f\left(a_{1}\right)+f\left(a_{2}\right)+\ldots+f\left(a_{k}\right) \geq f(A)
$$

thus at least one of $f\left(a_{i}\right) \geq\left(2^{k}-1\right) / k$, which was to be proven.

We show that apart from the $\log n$ factor, our result is the best possible. Namely, the method cannot give better lower bound than $n$ times the length of the secret.

Theorem 10. Given any access structure $\mathcal{A}$ on the n-element set $P$, we can always find a polymatroid function $f$ so that

(i) $f$ satisfies the conditions of Proposition 5;

(ii) $f(x) \leq n$ for all elements $x \in P$.

Proof. Let $A$ be a $k$-element subset of $P$, define

$$
f(A)=n+(n-1)+\ldots+(n+1-k) .
$$

This function assigns $n$ to each one-element set. If $A$ is a proper subset of $B$ then $f(B)-f(A)$ is the sum of $|B-A|$ consecutive positive integers, therefore it is $\geq 1$, and equality holds only if $B=P$ and $A$ is an $n-1$-element subset. This proves (i) of Proposition 5, and also proves the monotonicity of $f$. To check (ii), suppose that $A \cap B$ is a proper subset of both $A$ and $B$. Observe that the $(A \cup B)-A$ and $B-(A \cap B)$ is the same non-empty set, and suppose this difference contains, say $\ell \geq 1$ elements. Then both $f(A \cup B)-f(A)$ and $f(B)-f(A \cap B)$ is the sum of $\ell$ consecutive integers, and since $A \cup B$ has more elements than $B$, each number in the first sum is bigger than the corresponding number in the second sum. Thus

$$
f(A \cup B)-f(A)>f(B)-f(A \cap B),
$$

and since the values are integers, the difference between the two sides is at least 1 , as was required. 


\section{Conclusion and future work}

We have constructed an access structure $\mathcal{A}$ on $n$ elements so that any perfect secret sharing scheme must assign a share which is of size at least $n / \log n$ times the size of the secret. The best previous upper bound was 1.5 [4]. From the other size, for our access structure we can construct a scheme which, for each secret bit, assigns at most $n$ bits to each participant. This means that in this case the upper and lower bounds are quite close.

Recall that the access structure $\mathcal{A}$ is generated by the minimal subsets $U_{i}$ for $i=0,1, \ldots, 2^{k}-2$. Let $s$ be a secret bit, and for each $i$ pick $\left|U_{i}\right|$ random bits so that their mod 2 sum equal to $s$. Distribute these bits among the members of $U_{i}$. Each participant gets as many bits as many $U_{i}$ 's he or she is in, thus each share is at most $2^{k}-1 \leq n$ bits.

We have seen in Theorem 10 that using polymatroids we cannot prove essentially better lower bounds. For general access structures, however, the known general techniques produce exponentially large shares [10]. In order to turn the construction in Theorem 10 into an actual secret sharing scheme, and thus proving that every access structure can be realized within an $n$-factor blow-up in shares, the first obstacle is the following problem.

Problem 11. Can every polymatroid be represented as the entropy of appropriately chosen random variables?

An affirmative answer would help in completing the construction. However, intuition says that the answer is no [7], and sometimes the size of a share must be much larger. In this case we have to look after additional inequalities the entropy function does not share with polymatroids. These might help in establishing better lower bounds for the size of the shares.

\section{References}

1. G. R. Blakley and C. Meadows, Security of Ramp Schemes, Proceeding of Crypto'84 - Advances in Cryptology',Lecture Notes in Computer Science, Vol 196, G. R. Blakley and D. Chaum, eds. Springer-Verlag, Berlin, 1985, pp. 411-431.

2. C. Blundo, A. De Santis, L. Gargano, U. Vaccaro, On the Information Rate of Secret Sharing Schemes, in Advances in Cryptology - CRYPTO '92, Lecture Notes in Computer Science, Vol 740, E. Brickell ed, Springer-Verlag, Berlin, 1993, pp. 149-169.

3. C. Blundo, A. De Santis, A. G. Gaggia, U. Vaccaro, New Bounds on the Information Rate of Secret Sharing Schemes, Preprint, 1993

4. R. M. Capocelli, A. De Santis, U. Vaccaro, On the Size of Shares for Secret Sharing Schemes, Journal of Cryptology, Vol 6(1993) pp. 157-167.

5. M. Carpentieri, A. De Santis, U. Vaccaro, Size of Shares and Probability of Cheating in Threshold Schemes, Proceeding of Eurocrypt'93.

6. I. Csiszár and J. Körner, Information Theory. Coding Theorems for Discrete Memoryless Systems, Academic Press, New York, 1981.

7. I. Csiszár, personal communication. 
8. M. van Dijk, On the Information Rate of Perfect Secret Sharing Schemes, Proprint, 1994

9. S. Fujishige, Polymatroid dependence structure of a set of random variables, Information and Control 39(1978) pp. 55-72.

10. M. Ito, A. Saito, T. Nishizeki, Multiple Assignment Scheme for Sharing Secret Journal of Cryptology, Vol 6(1993) pp. 15-20.

11. K. Kurosawa, K. Okada, K. Sakano, W. Ogata, S. Tsujii, Nonperfect Secret Sharing Schemes and Matroids, Proceedings of Eurocrypt'93.

12. G. J. Simmons, An Introduction to Shared Secret and/or Shared Control Schemes and Their Application, Contemporary Cryptology, IEEE Press pp. 441-497, 1991.

13. E. Sperner, Ein Stas über Untermengen einer endlichen Menge, Math. $Z$. $27(1928)$, pp. 544-548. 